\title{
EL PLAGIO: PARTE DE NUESTRA CRUDA REALIDAD
}

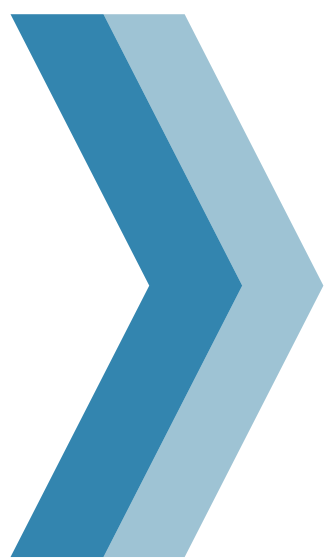

\section{PLAGIARISM: PART OF OUR RAW REALITY}

\section{Brito Ebert Falcón Guerrero"}

1. Doctor en estomatología. Especialista en periodoncia e implantología. Docente de la Universidad Nacional Jorge Basadre Grohmann.

\section{RESUMEN}

El plagio, en nuestra realidad, se está volviendo un mal endémico que no solo se aprecia en la vida cotidiana, sino también en las instituciones universitarias (tanto en pregrado como posgrado). Lamentablemente, en ciertas ocasiones se aprecia de forma circunstancial, dado que algunos autores (con un aparente descuido, anuencia o dejadez de sus asesores y/o jurados) tienden a buscar el facilismo del copiar y pegar, pasando desapercibidos y sin tener ningún tipo de sanción. La sanción se hace necesaria para así lograr erradicar estas malas prácticas en la difusión de las investigaciones. En tal caso, esta revisión tiene por objetivo evidenciar esta problemática y difundir las penalidades a las cuales pueden ser sujetos quienes la practiquen, así como de propiciar una tolerancia cero, ante esta situación.

Palabras clave: Ética, fraude, investigación, plagio, universidades (DeCS).

\begin{abstract}
Plagiarism in our reality is becoming an endemic evil that is not only seen in daily life, but also in university institutions (both undergraduate and postgraduate). Regrettably, in certain occasions it is appreciated circumstantially, since some authors (with an apparent carelessness, consent or neglect of their advisors and / or juries) tend to seek the easy way that implies to copy and paste. They usually go unnoticed and without having any type of sanction. Sanctioning is necessary in order to eradicate this bad practice in the dissemination of research. This review aims to highlight this problem and disseminate the penalties that may be applied, as well as encouraging zero tolerance in the face of this situation.
\end{abstract}

Keywords: Ethics, fraud, research, plagiarism, universities. 


\section{INTRODUCCIÓN}

Se ha vuelto cotidiano ver en las noticias denuncias públicas de plagio que se presentan en personajes ligados a la vida política e institucional. Del mismo modo, aunque no es de forma pública, esta problemática también se percibe en las instituciones universitarias. Es así que se señala que el plagio académico es una práctica demasiado frecuente entre los estudiantes universitarios ya que las nuevas tecnologías, y en especial, el internet permiten realizarlo con facilidad ${ }^{(1)}$.

Por ende, las ciencias de la salud no están exentas de presentar casos de plagio; por lo que, se debe hacer mayor énfasis sobre las implicancias y consecuencias de este acto que va en desmedro de la producción científica nacional.

La universidad como centro superior de estudios asume la alta responsabilidad histórico cultural de formar hombres capaces de producir, conservar y transmitir conocimiento científico-técnico y humanístico para el mejoramiento de la sociedad y la humanidad. Con el objetivo de cumplir con esta tarea, se ha valido y seguirá valiendo de variadas y novedosas maneras en el tiempo. La investigación se perfila como la forma más emblemática y tradicional, ya que tanto científica como humanística es el fruto maduro más valioso en su esencia ${ }^{(2)}$.

Es importante promover dentro de las instituciones universitarias una correcta adquisición del conocimiento; por tanto, se debe disponer de buenos modelos docentes. Sierra y col. ${ }^{(3)}$ cita a Gusdorf, quien manifiesta que "el maestro es aquel que da ejemplo, pero buen ejemplo". En tal razón, es muy importante poner mayor énfasis en la calidad de formación de los docentes universitarios, tanto ética como académicamente.

Si bien es cierto, todo lo aprendido gracias a la formación y educación ya ha sido propuesto por otras personas, debemos tener en cuenta que en un trabajo de investigación es necesario realizar una búsqueda bibliográfica vigente y actualizada, pero esta búsqueda solo debe servir para proporcionarnos la información necesaria para establecer una idea original y una nueva propuesta de investigación.

El objetivo de este artículo es evidenciar, en base a los antecedentes científicos, esta problemática y difundir las penalidades a las cuales pueden ser sujetos quienes la practiquen.

\section{PLAGIO}

Según la Real Academia Española de la Lengua, la definición formal de plagio es la siguiente:

Plagio. (Del lat. plagium).1. m. Acción y efecto de plagiar (copiar obras ajenas).2. m. Am. Acción y efecto de plagiar (secuestrar a alguien).

Plagiar. (Del lat. plagiāre).1. tr. Copiar en lo sustancial obras ajenas, dándolas como propias (RAE, s.f.).

Contrariamente a lo que algunos podrían pensar, el plagio no es un problema nuevo ya que, según cuenta Vitrubio, en el siglo $V$ a.c, varias obras antiguas de la biblioteca de Alejandria fueron presentadas durante un concurso de poesía por varios concursantes que se atribuían así mismos dichas obras. Seguidamente, los participantes al ser descubiertos fueron castigados como ladrones según las leyes de esa época ${ }^{(4)}$.

El plagio es una forma de mala conducta en la investigación y una grave violación de las normas de la ciencia. Es la tergiversación de las ideas o palabras de otro como propias, sin el debido reconocimiento de la fuente original. Ciertos aspectos del plagio lo hacen menos directo de lo que sugiere esta definición ${ }^{(5)}$.

Helgesson y Eriksson ${ }^{(6)}$ mencionan que el plagio se compone de dos partes: La primera parte es la apropiación del trabajo de otra persona, y la segunda consiste en pasar el trabajo como propio al no dar el crédito adecuado al autor original.

\section{PENALIDADES}

La figura del plagio se ha extendido con la aparición del Internet, sin embargo, las denuncias son muy escasas en nuestro país, pero existen bases legales que sancionan este mal proceder. En el Perú, la base legal se encuentra en las normas siguientes:

a) Constitución Política del Perú de 1993. Artículo 2o: Toda persona tiene derecho a: [...] 8. A la libertad de creación intelectual, artística, técnica y científica, así como a la propiedad sobre dichas creaciones y a su producto. El Estado propicia el acceso a la cultura y fomenta su desarrollo y difusión.

b) Código Penal (2007). Artículo $219^{\circ}$ : Será reprimido con pena privativa de libertad no menor de cuatro ni mayor de ocho años y noventa a ciento ochenta días 
multa; el que, con respecto a una obra, la difunda como propia, en todo o en parte, copiándola o reproduciéndola textualmente, o tratando de disimular la copia mediante ciertas alteraciones, atribuyéndose $o$ atribuyendo a otro la autoría o titularidad ajena.

c) Código Civil (2008). Artículo $18^{\circ}$. Protección de los derechos de autor e inventor. Los derechos del autor o del inventor, cualquiera sea la forma o modo de expresión de su obra, gozan de protección jurídica de conformidad con la ley de la materia.

d) Decreto Legislativo Núm. 822 Ley sobre el Derecho de Autor ${ }^{(7)}$.

Estas normas son importantes para difundir, educar y disuadir a los estudiantes de cometer el plagio, ya que algunos estudios han demostrado que la "aplicación de sanciones disuaden efectivamente a los estudiantes a querer plagiar" ${ }^{(8)}$.

\section{FORMAS DE PLAGIO}

De García ${ }^{(7)}$ cita a Tripathi y Kumar para referirse a las formas de plagio. El autor señala que estos autores "[...] han elaborado una de las clasificaciones del plagio más completas, pues enumeran diecisiete tipos de plagio $[\ldots]$," los cuales son presentados a continuación.

\section{Sin cita de fuentes:}

1.1 Entregar un trabajo ajeno como si fuera propio; 1.2 Reproducir fragmentos completos sin alteración; 1.3 Combinar distintas fuentes sin referenciar ninguna; 1.4 Reproducir un fragmento, del que se cambian ciertas palabras; 1.5 Reformular distintas fuentes que conforman el trabajo final; 1.6 "Préstamo" del trabajo propio anterior, con el que se viola requisitos de originalidad.

\section{Con fuentes citadas (pero aun así plagiadas):}

2.1 Se cita al autor, pero sin los datos necesarios para localizar la fuente. La práctica suele esconder otras formas de plagio. 2.2 No se puede localizar las fuentes citadas debido a su referencia incorrecta. 2.3 Se cita al autor, pero no se entrecomilla las palabras textuales, por lo que se hace pasar por propia una interpretación ajena. 2.4 Las citas y referencias son correctas, pero prácticamente constituyen la mayor parte del artículo. 2.5 En algunos fragmentos se citan de manera correcta las fuentes, pero en otros se introduce una paráfrasis de esas mismas fuentes, esta vez sin reconocimiento expreso.

\section{Otros tipos de plagio:}

3.1 Reproducir citas directas sin comillas. 3.2 Cambiar algunas palabras de la cita, que se reproducen sin comillas. 3.3 Emplear lenguaje creativo de un autor sin hacer constar el debido reconocimiento. 3.4 Hacer pasar una idea ajena como propia. 3.5 Seguir el razonamiento de otro autor en un orden aproximado al que se desarrolla en el artículo original. 3.6 Copiar datos procedentes de otros estudios.

Miyahira ${ }^{(9)}$ se pregunta ¿Qué hacer para prevenir el plagio?, respondiéndose que "es obligación y responsabilidad de las universidades hacer todos los esfuerzos posibles para prevenir la ocurrencia de plagio. Una de las acciones sería exigir a los docentes el uso de técnicas de enseñanza-aprendizaje que obliguen al estudiante al análisis de la información, más que la sola copia, y capacitar a los docentes y alumnos en las formas apropiadas de hacer las citas bibliográficas".

En tal sentido, Huamani y col. ${ }^{(10)}$ describen una alta frecuencia de copia en investigaciones durante el pregrado provenientes de fuentes electrónicas, por lo que es necesario prevenir que se repita en futuras investigaciones al implementar sistemas de búsqueda sistemática de plagio. Proponiendo un proceso de búsqueda adaptada de "copia y pega" en trabajos de investigación, para detectar el plagio:

1. Identificar las oraciones del texto de la introducción (no se incluyen definiciones o términos de uso sin restricción).

2. Buscar en Google: Para esto se "copia" literalmente la oración y se "pega" en el buscador sin usar " "(Ejm: oración escrita en la introducción, aquí no excluimos los conectores).

3. De no encontrar resultados coincidentes, la oración es modificada eliminando los conectores, permaneciendo sólo las palabras que constituyen la oración (Ejm: oración escrita introducción excluimos conectores).

4. Verificar los resultados del buscador: se identifican las fuentes de "copia" si la coincidencia es literal o parcial con la oración introducida.

5. Calificar la proporción de la "copia": se señala el número de oraciones copiadas y calidad de la fuente electrónica utilizada.

Estas herramientas son importantes de tener en cuenta, debido a que existen antecedentes que demuestran una alta frecuencia de plagio en las tesis analizadas en la universidad pública peruana ${ }^{(11)}$. 
Se debería seguir el ejemplo del gobierno federal de EE.UU, quien ha desarrollado y perfeccionado sus políticas sobre mala conducta. Las agencias federales, así como las instituciones de investigación, han establecido enfoques para responder a las denuncias e instancias de plagio ${ }^{(5)}$.

Recientemente, Rathore ${ }^{(12)}$ recomienda que se deberían realizar talleres focalizados sobre cómo detectar y evitar la mala conducta científica. Esto, ayudaría aumentar el conocimiento y fortalecer las actitudes que eviten el plagio.

Moran ${ }^{(2)}$ comparte páginas y motores de búsqueda que pueden facilitar la detección de un posible plagio, usando una determinada cadena de palabras del texto sospechoso. Esto con el fin de ver si se encuentra un texto potencialmente plagiado:

- http://www.turnitin.com- Permite comparar trabajos de estudiantes entre ellos mismos y con artículos disponibles en línea. Ofrece un "trial" de un mes.

- http://www.plagiarismdetect.com/- Es un servicio gratuito que permite identificar textos que han sido plagiados.

-http://www.plagiarism.phys.virginia.edu/Wsoftware. html - WCopyfind es un programa gratuito para detectar plagio.
-http://www.plagiarism.com/-La compañía Glatt Plagiarism Services, Inc. produce tres diferentes programas que ayudan a detectar y disuadir el plagio.

- http://www.canexus.com/-Eve2 hace una búsqueda bastante exhaustiva. Informa que porcentaje del ensayo fue plagiado y coloca en color rojo las partes plagiadas.

- https://.ipd.uni-karlsruhe.de/jplag/- Plag es un sistema que encuentra similitudes entre varios archivos.

- http://www.copyscape.com/- Es un servicio que ayuda a los autores a detectar cuando el contenido de sus "sites" es plagiado. Existe una versión gratuita.

- http://www.articlechecker.com/- Servicio gratuito para detección de plagio.

Finalmente, se concluye que debería haber tolerancia cero para evitar el plagio; por lo que siempre se debe de contar con el apoyo de programas de detección actualizados y difusión de las sanciones y penalidades para lograr concientizar a los investigadores que promueven y realicen el plagio; considerando como único fin mejorar la originalidad de los trabajos de investigación en las ciencias de la salud.

\section{REFERENCIAS BIBLIOGRÁFICAS}

1. Alfaro Torres $P$, de Juan Juárez $\mathrm{T}$. El plagio académico: formar en competencias y buenas prácticas universitarias. RUIDERAe: Revista de Unidades de Información. Número 6 ( 2 o semestre 2014 ). Disponible en: https://revista.uclm.es/index.php/ruiderae/article/downloa d/637/538.

2. Morán Seminario HM. El plagio en la vida académica universitaria. Universidad Ricardo Palma. Disponible en: ht t p://www.urp.edu. pe/vicerrectorado-deinvestigacion/download.php?archivo $=\mathrm{cmVwb3NpdG9yaW9}$ cQXJ07WN1bG9zIEFjYWTpbWljbyB5IGRIIEludmVzdGInYWN p825cTW9y4W4gSOljdG9yIEVsIFBsYWdpbyBIbiBsYSBWaW RhIEFjYWTpbWIjYSBVbmI2ZXJpc3RhcmIhIDIwMTYtSUkucG Rm.

3. Sierra B, Arrieta A. El papel del ejemplo en la formación de profesores. 1999. Aula abierta No 73. Disponible en: https://dialnet.unirioja.es/descarga/artículo/45448.pdf.

4. Soto Rodríguez A. El plagio y su impacto a nivel académico y profesional. E-Ciencias de la Información. Volumen 2, numero 1, articulo 2, ene-jun 2012.

5. Anderson MS, Steneck NH. Seminar article-The problem of plagiarism. Urologic Oncology: Seminars and Original Investigations. 2011;29:90-94.
6. Helgesson G, Eriksson S. Plagiarism in research.Med Health Care and Philos. 2015;18:91-101.

7. De García RQP. Sanción al plagio de obras literarias en el Instituto Nacional de Defensa de la Competencia y de la Protección de la Propiedad Intelectual (Indecopi) en Perú. Investigación bibliotecológica. 2014;28 (63): 115-162.

8. Bilic-Zulle L, Azman J, Frkovic V. Mladen Petroveckils There an effective approach to deterring students from plagiarizing?. Sci Eng Ethics. 2008; 14:139-147.

9. Miyahira JM. Editorial-Plagio como mala Conducta Académica. Acta Herediana octubre. 2015 - marzo 2016; Vol. 57:5-7.

10. Huamaní C, Dulanto-Pizzorni A, Rojas-Revoredo V. 'Copiar y pegar' en investigaciones en el pregrado: haciendo mal uso del Internet. An Fac med. 2008; 69(2):117-9.

11. Saldaña-Gastulo JJC, Quezada-Osoria CC, Peña-Oscuvilca A, Mayta-Tristán P. Alta frecuencia de plagio en tesis de medicina de una universidad pública peruana. Rev Peru Med Exp Salud Publica. 2010; 27(1): 63-67.

12. Rathore F A, Fatima N E, Farooq F, Mansoor SN. Combating scientific misconduct: the role of focused workshops in changing attitudes towards plagiarism. Cureus (May 28, 2018). 10(5): e2698. DOI 10.7759/cureus.2698.

\section{Correspondencia}

Britto Ebert Falcón-Guerrero

E-mail: artdent2000@hotmail.com
Fecha de recepción: 04 de octubre 2018

Fecha de aceptación: 22 de marzo de 2019 